\title{
Ligand-Accelerated Cu-Catalyzed Azide-Alkyne Cycloaddition: a Mechanistic Report
}

Valentin O. Rodionov, Stanislav Presolski, David D. Díaz, Valery V. Fokin, M.G. Finn* Department of Chemistry and The Skaggs Institute of Chemical Biology The Scripps Research Institute, 10550 N. Torrey Pines Rd., La Jolla, CA 92037, USA

\section{Supporting Information}
A. General S1
B. Kinetics Data S2
1. Methods $\mathrm{S} 2$
2. Data for Table 1 $\mathrm{S} 2$
3. Data for Figure 2B S5
4. Observation of two kinetic pathways at low conversion S8
5. Hammett study using $p$-substituted arylazides $\mathrm{S} 10$
C. References S11

\section{A. General}

Reagents and solvents were purchased from VWR or Acros, and were used as received. Ligand 4 was obtained from Aldrich. Aromatic azides were obtained either by diazotization of the corresponding anilines, ${ }^{1}$ or by the convenient copper-catalyzed reaction ${ }^{2}$ from the corresponding aryl bromides. THF, acetonitrile, diethyl ether, dichloromethane, and toluene were dried by passage through activated alumina columns. Chromatography was performed using SINGLE StEP pre-packed MPLC columns (Thomson Instrument Company, Oceanside, CA). ${ }^{1} \mathrm{H}$ and ${ }^{13} \mathrm{C}$ NMR spectra were recorded on a Varian $\mathrm{AM} 200$ spectrometer in $\mathrm{CDCl}_{3}, \mathrm{CD}_{3} \mathrm{OD}$, $\mathrm{CD}_{3} \mathrm{CN}$ or DMSO- $d_{6}$. Routine mass spectra were obtained using an Agilent 1100 (G1946D) ESI MSD with mobile phase composed of 9:1 $\mathrm{CD}_{3} \mathrm{CN}: \mathrm{H}_{2} \mathrm{O}$ containing $0.1 \% \mathrm{CF}_{3} \mathrm{CO}_{2} \mathrm{H}$. GC-MS analyses were performed on an HP GCD-II (Model 5810) instrument. Elemental analyses were performed by Midwest MicroLab, LLC. Infrared spectra were recorded on MIDAC-FTIR spectrometer in $\mathrm{KBr}$ pellets. The following stain solutions were used in addition to UV light with fluorescent TLC plates: phosphomolybdic acid, anisaldehyde/EtOH, and $\mathrm{KMnO}_{4} / \mathrm{H}_{2} \mathrm{O}$. Reactions requiring anhydrous conditions were performed under nitrogen.

WARNING: Low molecular weight azides such as the aryl azides used in this study are potentially explosive. Appropriate protection measures should always be taken when handling these compounds, the most important of which is to never isolate them away from solvent. 


\section{B. Kinetics data}

\section{Experimental Method}

The experimental procedure and second-order data treatment used is described in the Supporting Information to the accompanying article in this issue.

\section{Data for Table 1.}

Figure S1 (Table 1, entries 1-4) Conditions: 4:1 DMSO:Tris (133 mM), $25 \mathrm{mM}$ sodium ascorbate (pH 8.0 ). The left plot of each row is the raw data, and the middle is the plot of $\ln$ (rate) vs. $\ln$ (concentration) for rate constants determined by second-order fits to all of the data points on the left. The quality of these fits was generally good. The right plot of each row is the $\ln$ (rate) vs. $\ln$ (conc.) plot using first-order rate constants obtained with the data obtained at low conversion (the portion of the raw data that is linear starting at time $=0$ ). These are shown for comparison only; the values reported in the table derive from the more appropriate second-order fits.
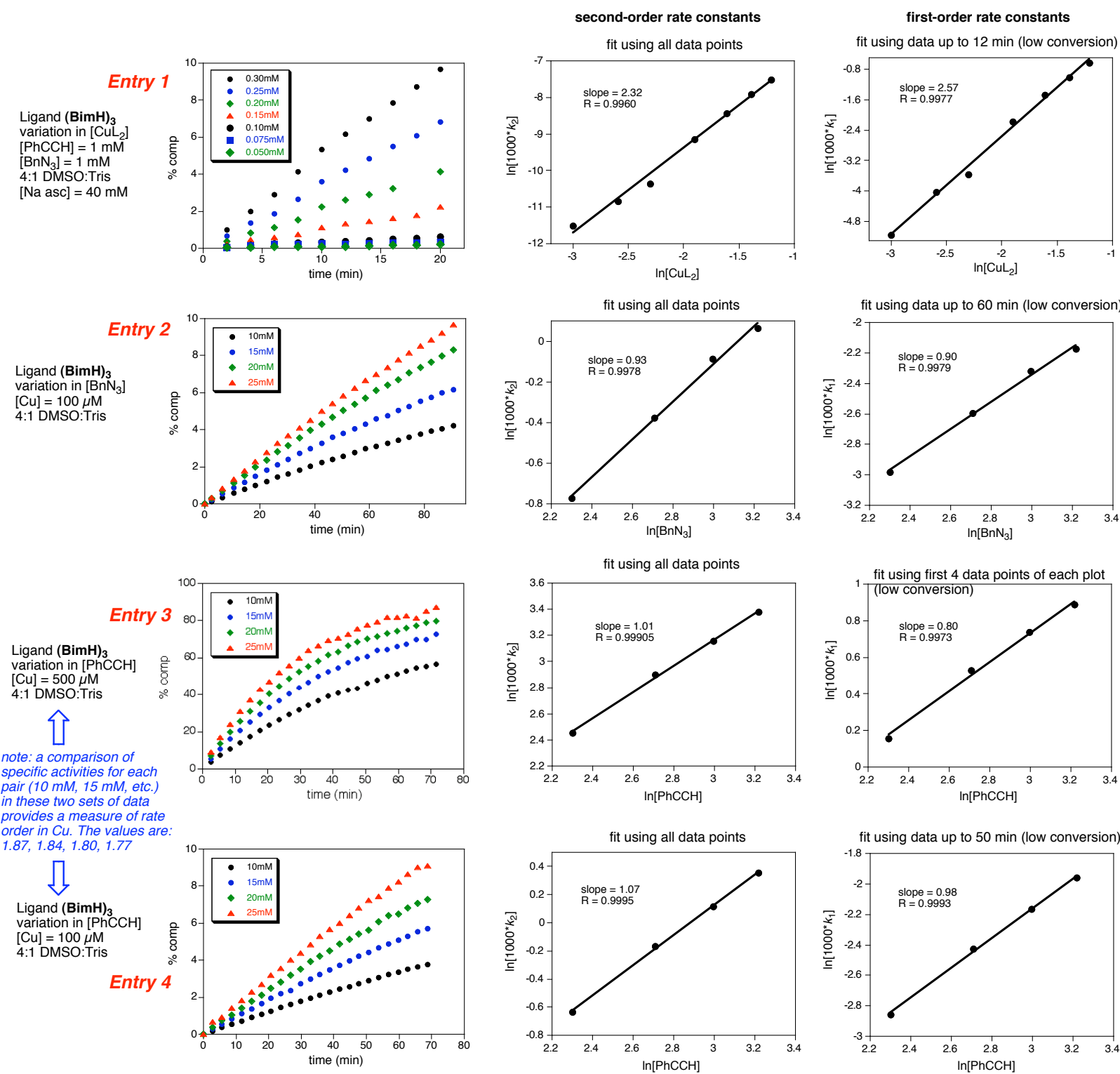
Figure S2 (Table 1, entries 5-6). Conditions: 4:1 DMSO:water, containing $25 \mathrm{mM}$ sodium ascorbate (pH 8.0). The plot at the right shows that 2,6-lutidine, a common additive to $\mathrm{CuAAC}$ reactions in organic solvents, does not change the threshold behavior of $\mathrm{Cu} \cdot(\mathbf{B i m H})_{3}$.
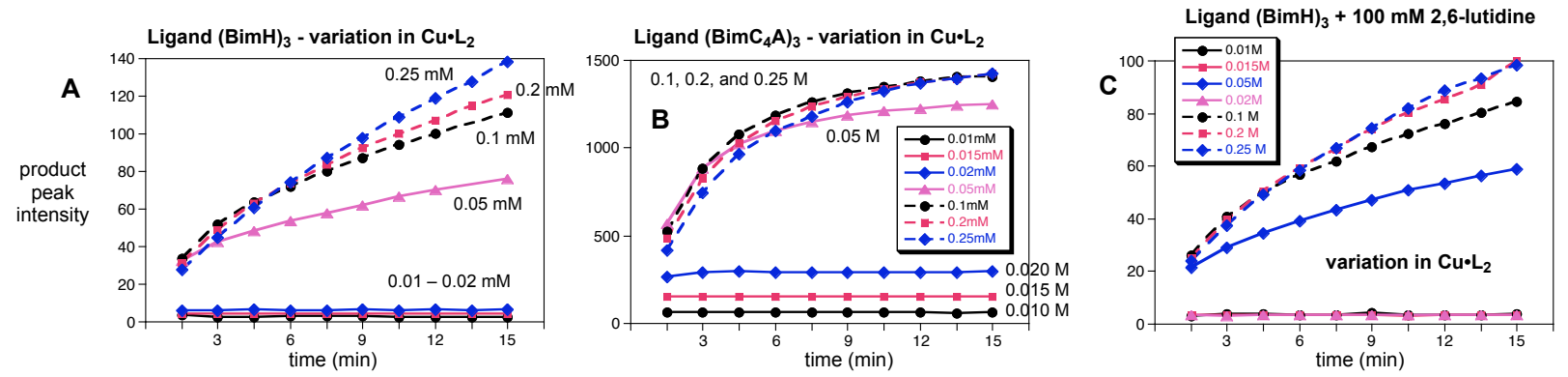

Figure S3 (Table 1, entries 7-8). Conditions: 4:1 DMSO:water, containing $25 \mathrm{mM}$ sodium ascorbate (pH 8.0). The left plot of each row is the raw data; the middle is the plot of $\ln ($ rate) vs. $\ln$ (concentration) for rate constants determined by second-order fits to all of the data points on the left. The quality of these fits was generally on the same order as those shown above, which is to say, quite good. The right plot in the first row is the $\ln$ (rate) vs. $\ln$ (conc.) plot using first-order rate constants obtained with the data obtained at low conversion (the portion of the raw data that is linear starting at time $=0$ ). This is shown for comparison only.
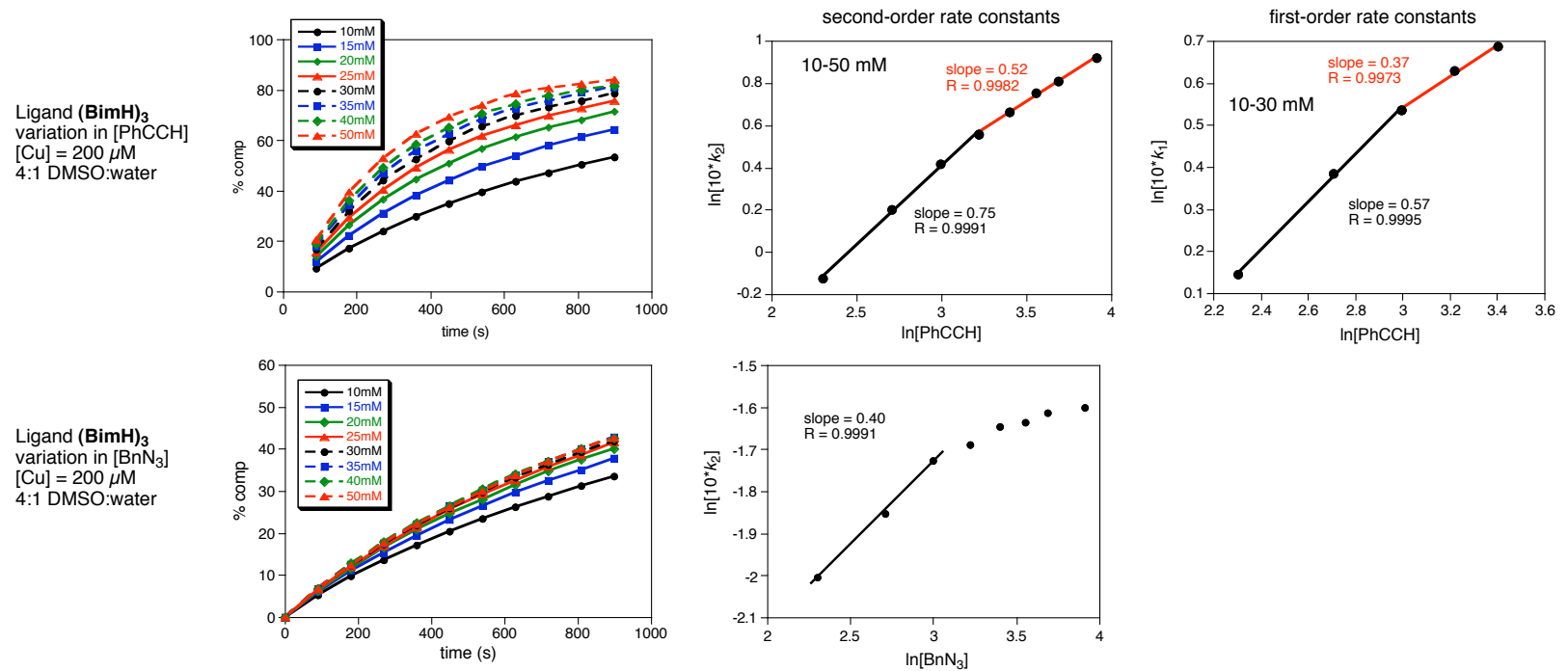
Figure S4 (Table 1, entries 9-10). Conditions: 4:1 DMSO:aq. $\mathrm{KCl}(133 \mathrm{mM})$, containing $25 \mathrm{mM}$ sodium ascorbate $(\mathrm{pH} \mathrm{8.0)}$. The left plot of each row is the raw data; the middle is the plot of $\ln (\mathrm{rate})$ vs. $\ln$ (concentration) for rate constants determined by second-order fits to all of the data points on the left. The quality of these fits was generally on the same order as those shown above, which is to say, quite good. The right plot of each row is the $\ln$ (rate) vs. $\ln$ (conc.) plot using first-order rate constants obtained with the data obtained at low conversion (the portion of the raw data that is linear starting at time $=0$ ). These are shown for comparison only; the values reported in the table derive from the more appropriate second-order fits.
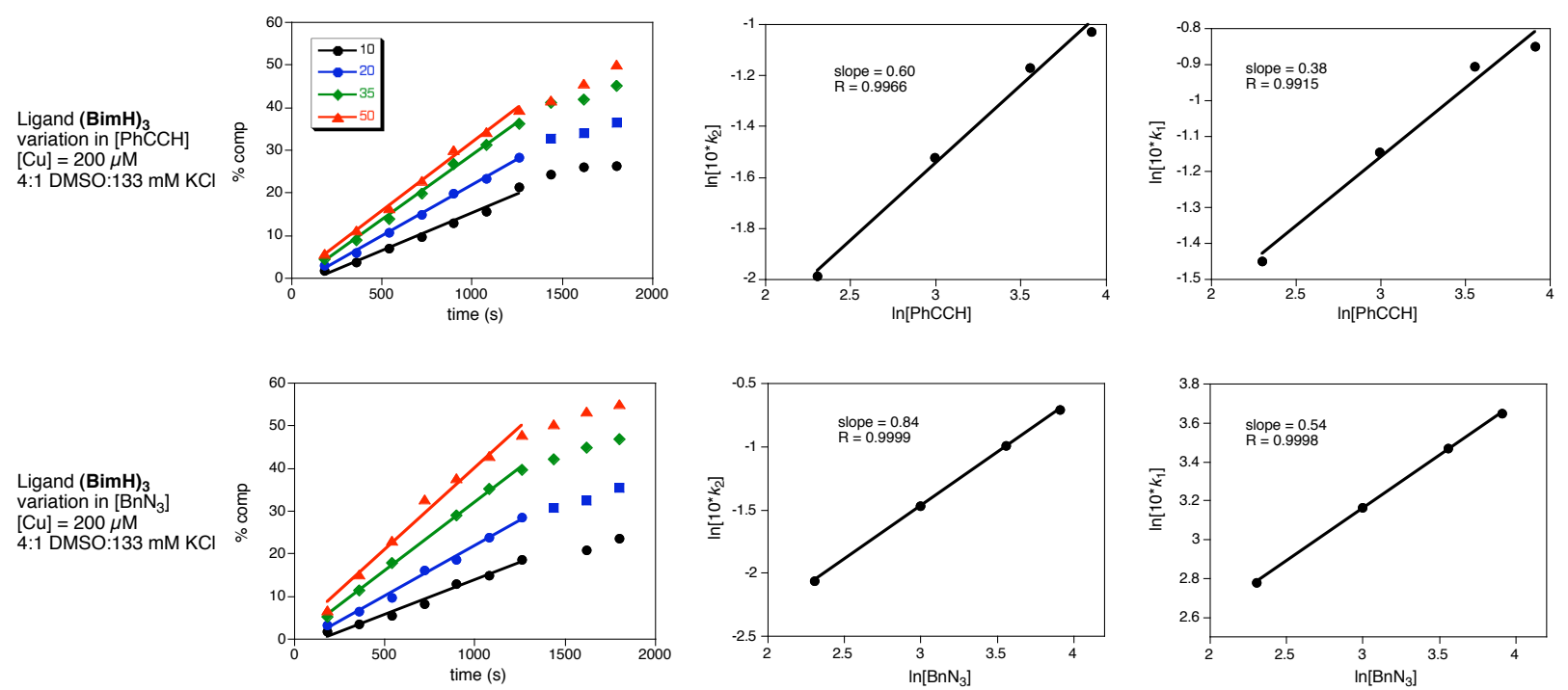

Figure S5 (Table 1, entries 11-12). Conditions: 4:1 DMSO:water, containing $25 \mathrm{mM}$ sodium ascorbate $(\mathrm{pH}$ 8.0). The left plot of each row is the raw data; second- and first-order treatment of the second of these is as described in the figures above.
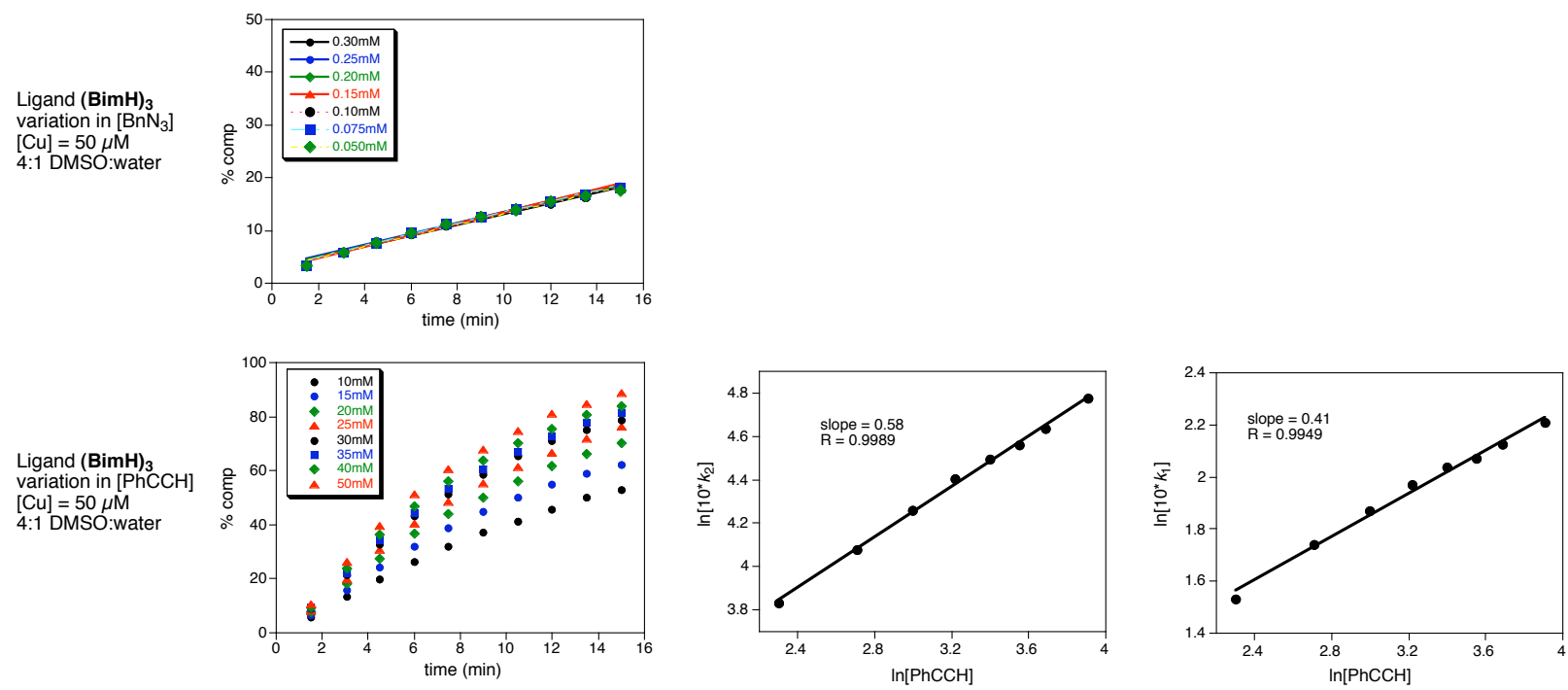
Figure S6 (Table 1, entries 13-15). Conditions: 4:1 DMSO:water, containing $25 \mathrm{mM}$ sodium ascorbate (pH 8.0).
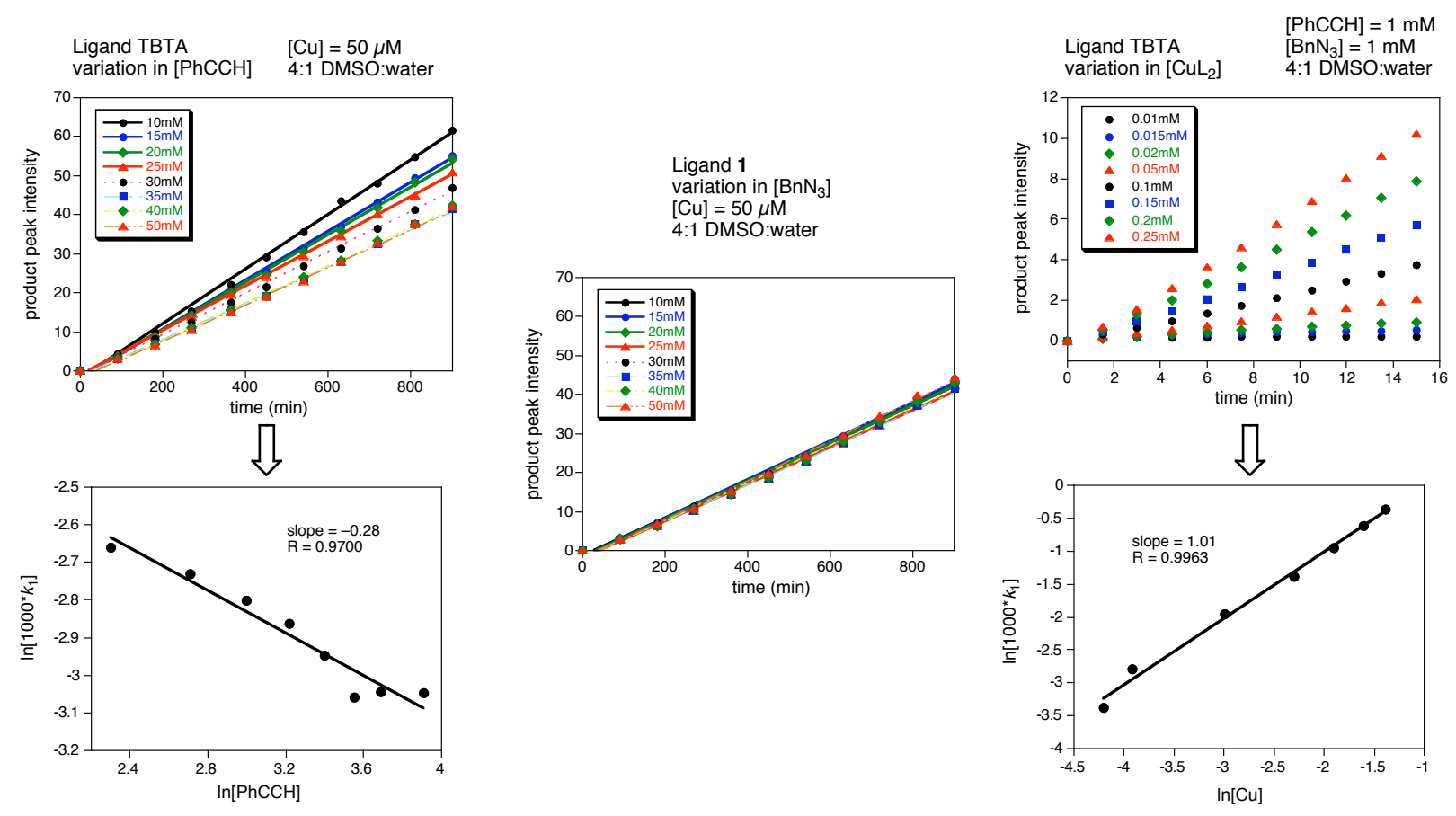

3. Data for Figure 2B: relative rates for production of monotriazole 2 and bis-triazole 3 from 1.

The initial portion of each of the kinetic runs was subjected to the second-order kinetic analysis described in the preceding paper, resulting in the plots shown below. Many of the fits are only approximate, since the production of neither of the products is likely to be a secondorder process. The values therefore represent only a convenient way to compare each ligand to the others; the observed trend is the same for a pseudo-first-order treatment of the data at very low conversion. Note that for several of the more active catalysts, the production of monotriazole levels off midway through the reaction. 
Figure S7 (Figure 2B, monotriazole production from 1 + phenylacetylene). Conditions: $2 \mathrm{mM}$ diazide 1, $2 \mathrm{mM} \mathrm{PhCCH}, 0.1 \mathrm{mM} \mathrm{CuSO}_{4}, 0.2 \mathrm{mM}$ ligand, 4:1 DMSO: $\mathrm{H}_{2} \mathrm{O}$, containing $20 \mathrm{mM}$ sodium ascorbate, 24 ${ }^{\circ} \mathrm{C}$.
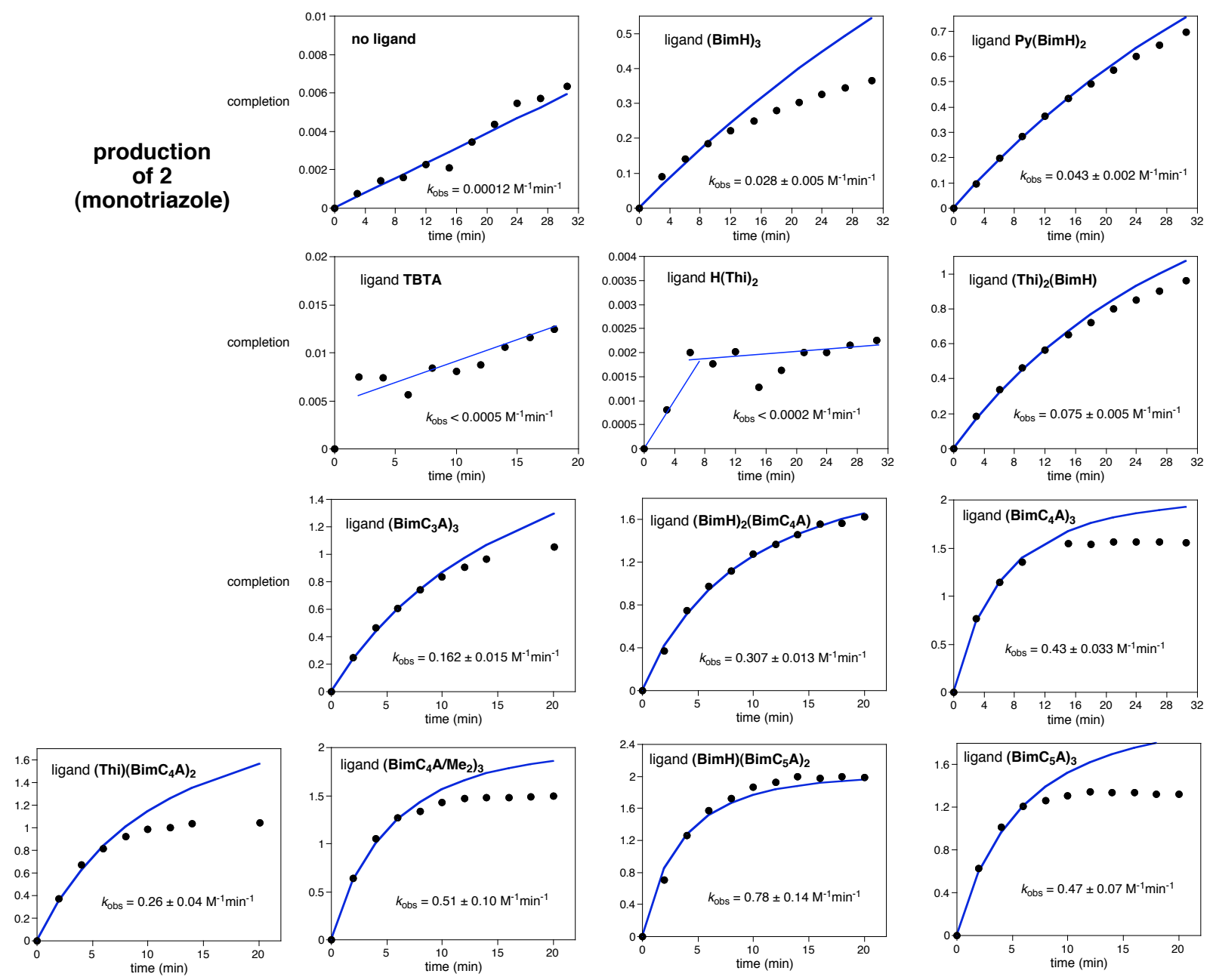
Figure S8 (Figure 2B, monotriazole production from 1 + phenylacetylene).
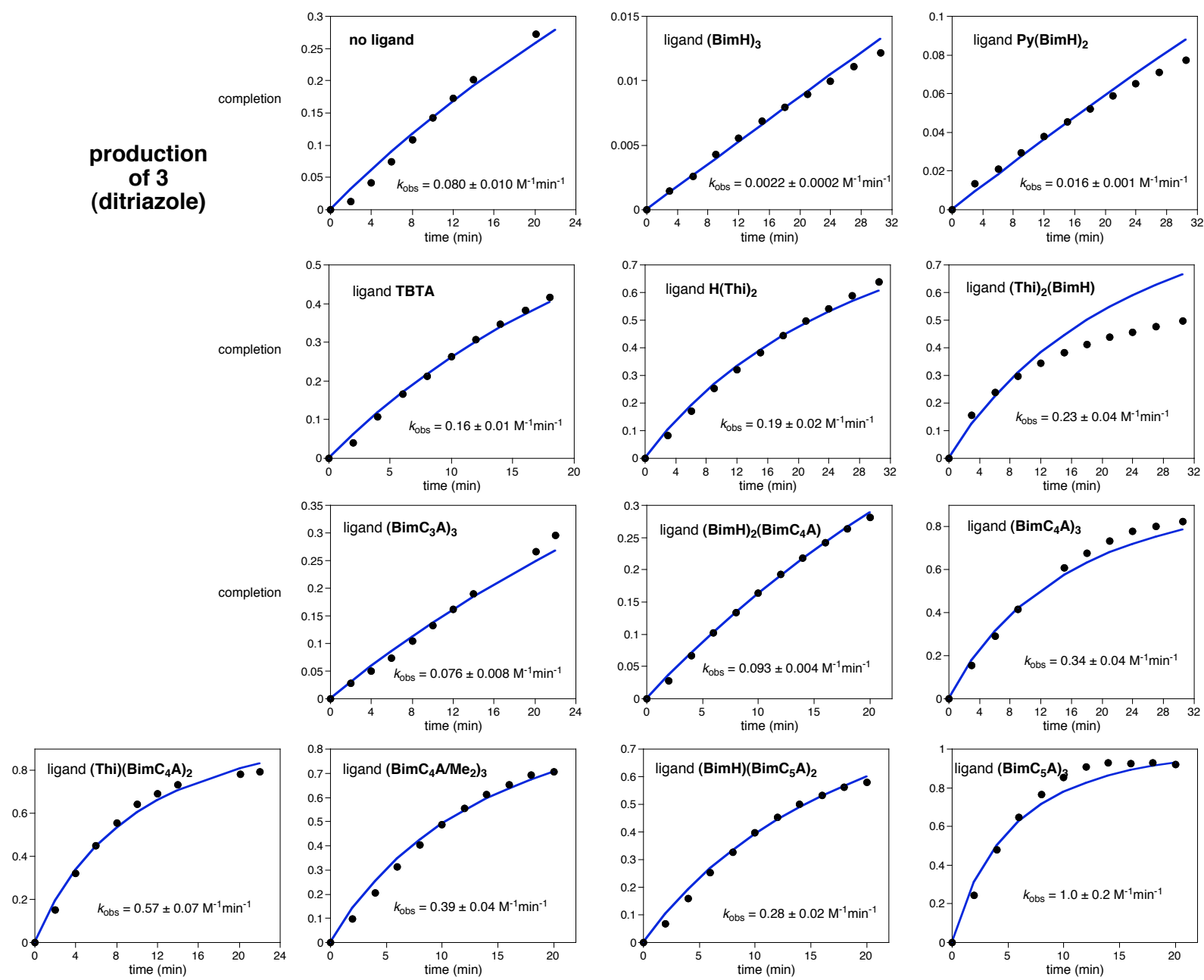
Figure S9 (Figure 2B, plots of $[\mathbf{2}+3]$ production from $1+$ phenylacetylene).
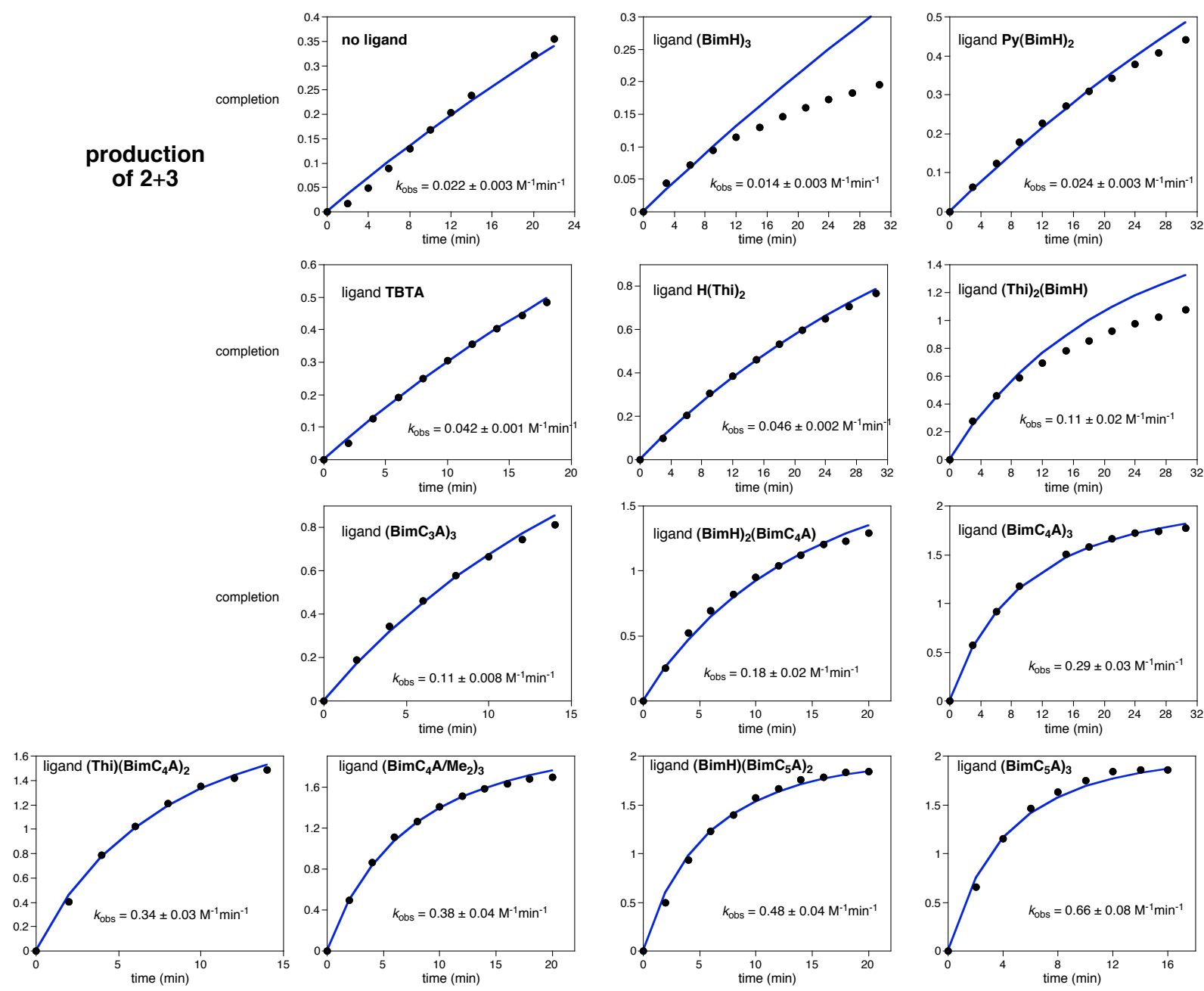

\section{Observation of two kinetic pathways at low conversion.}

Figure S10 below shows an interesting variation in reaction rate at the "threshold" $\mathrm{Cu}$ concentration of $50 \mu \mathrm{M}$. As decreasing amounts of ligand were added, the amount of product produced before the first aliquot increased. At the highest ligand:Cu ratio (5:1), the initial "burst" phase was completely suppressed, and the product-vs-time curve extrapolates to the origin. At the other extreme (ligand: $\mathrm{Cu}=0.4: 1$ ), approximately three times as much triazole was formed by the time the first aliquot is taken, representing approximately 30 equivalents with respect to $\mathrm{Cu}$. 
Figure S10. Product-vs-time data as a function of changes in $(\mathbf{B i m H})_{3}: \mathrm{Cu}$ ratio at $[\mathrm{Cu}]=50 \mu \mathrm{M}$. (Left) complete data; (middle) blowup of the first data point showing the increasing amounts of product formed by the time the first aliquot was taken at $120 \mathrm{~s}$; (right) second-order rate constants obtained from fitting of all the data points after normalizing each set to the same starting point at $t=120 \mathrm{~s}$, showing that the rate after this point was the same for each reaction.
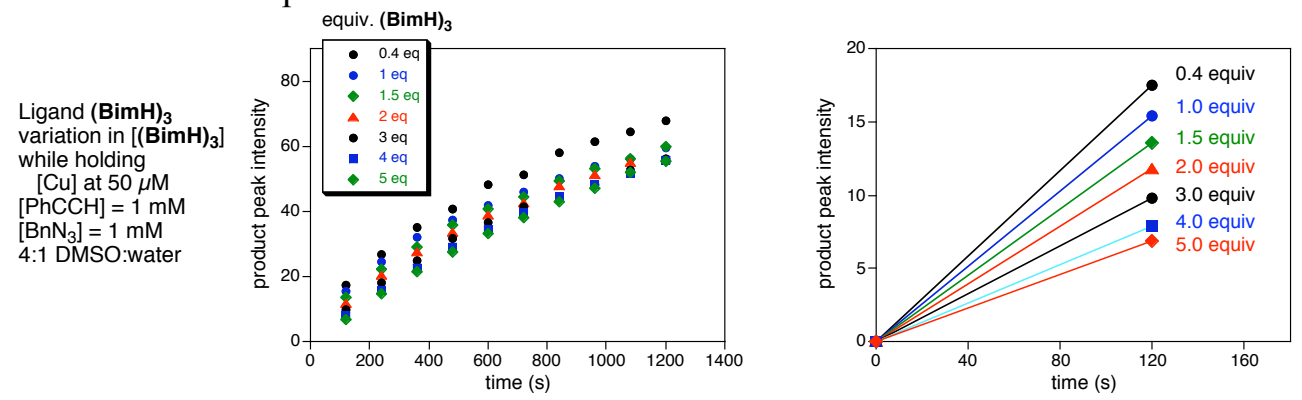

from second-order fits $k_{2}\left(\mathrm{M}^{-1} \mathrm{~s}^{-1}\right)$ after $\mathrm{t}=120 \mathrm{~s}$

0.4 equiv $=0.098 \pm 0.010$

1.0 equiv $=0.084 \pm 0.004$

1.5 equiv $=0.086 \pm 0.006$

2.0 equiv $=0.085 \pm 0.007$

3.0 equiv $=0.085 \pm 0.007$

4.0 equiv $=0.087 \pm 0.009$

5.0 equiv $=0.086 \pm 0.010$

Figure S11. Product-vs-time data for different order of addition of reaction components at relatively low $\mathrm{Cu}$ concentration in the presence of ligand $\left(\mathrm{BimC}_{\mathbf{4}} \mathbf{A}\right)_{3}$. The reagent added last is indicated in the plot legend. $[\mathrm{Cu}]=20$ $\mu \mathrm{M} ;[\mathrm{PhCCH}]=\left[\mathrm{BnN}_{3}\right]=1 \mathrm{mM} ;[\mathrm{Na}$ ascorbate $]=25 \mathrm{mM} ;\left[\left(\operatorname{BimC}_{4} \mathbf{A}\right)_{3}\right]=$ $40 \mu \mathrm{M}$, except for the runs marked "Asc. (0.25)" and "Asc. (0.5)," for which the concentration of the ligand was $5 \mu \mathrm{M}$ and $10 \mu \mathrm{M}$, respectively.

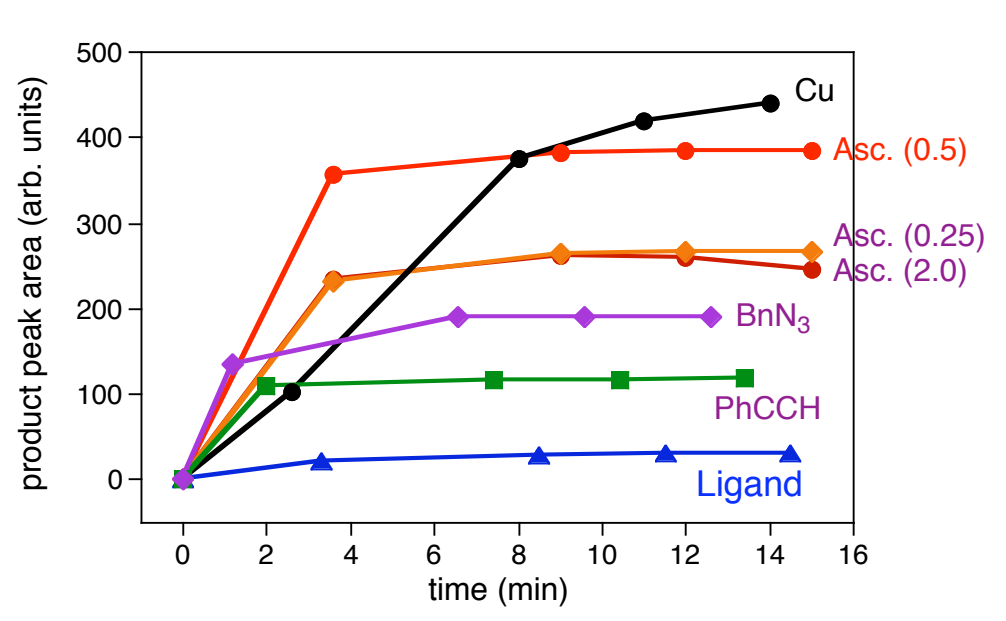

Figure S11 shows that the order of addition makes a difference, in this case for a reaction done with $20 \mu \mathrm{M} \mathrm{Cu}$, which is below the threshold that allows for a sustained reaction. The addition of ascorbate last to initiate the reaction provides for the largest "burst" before catalysis stops, even in the presence of varying amounts of ligand with respect to metal. The addition of azide or alkyne last provides for fewer turnovers, and the addition of ligand last is unable to rescue the slowly-reacting catalyst from a premature death. When $\mathrm{Cu}$ is added last, the system gives slower initial reaction, but this lasts longer before tapering off.

Ligand complexation clearly preserves active $\mathrm{Cu}$, even under reducing conditions. Alkyne, a strongly coordinating component relative to azide, likewise seems to be protective; azide is perhaps less so. The longest-lived systems are observed when all the coordinating ligands are present before the initiation of the reaction, either by addition of $\mathrm{Cu}$ or reducing agent. Such results could not occur if ligand exchange on $\mathrm{Cu}(\mathrm{I})$ (including ligand substitution reactions, disproportionation/comproportionation processes, exchange of cluster components, etc.) were fast with respect to turnover frequency. In other words, these data strongly support the notion that copper is slow to adjust its coordination and/or aggregation state relative to the rate of the $\mathrm{CuAAC}$ reaction, and therefore that multiple active and inactive species may be present. 


\section{Hammett study using p-substituted aryl azides.}

Figure S12. Calibration of aryltriazoles vs. internal standard (1-benzyl-4-phenyl-1,2,3-triazole- $d 7$ ). Individual calibration runs made with independent solutions are plotted, with the results of curve fitting (linear or binomial) shown below each plot. Above each plot is the curve fit to the data taken all together; these values were used to convert peak intensities to concentration for second-order kinetics.

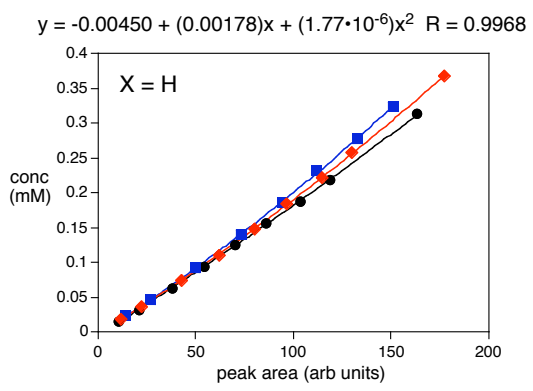

$y=-0.004373+(0.001744) x+\left(1.18 \cdot 10^{-6}\right) x^{2} \quad R=0.9999$ $y=-0.002866+(0.001766) x+\left(2.65 \cdot 10^{-6}\right) x^{2} R=0.9999$ $y=-0.002468+(0.001708) x+\left(2.15 \cdot 10^{-6}\right) x^{2} \quad R=0.99995$

$y=-0.002059+(0.001469) x+\left(5.62 \cdot 10^{-7}\right) x^{2} R=0.9989$

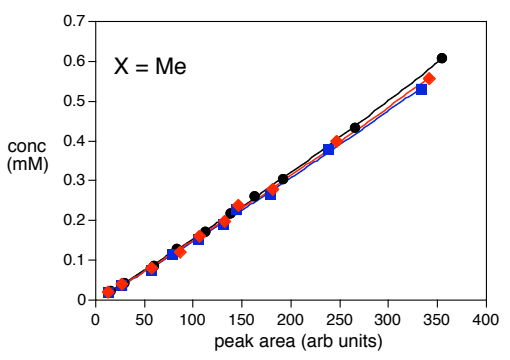

$y=-0.00372+(0.001477) x+\left(6.55 \cdot 10^{-7}\right) x^{2} \quad R=0.9999$ $y=-0.003227+(0.001465) x+\left(4.46 \cdot 10^{-7}\right) x^{2} \quad R=0.9992$ $y=-0.003863+(0.001501) x+\left(4.36 \cdot 10^{-7}\right) x^{2} R=0.9993$

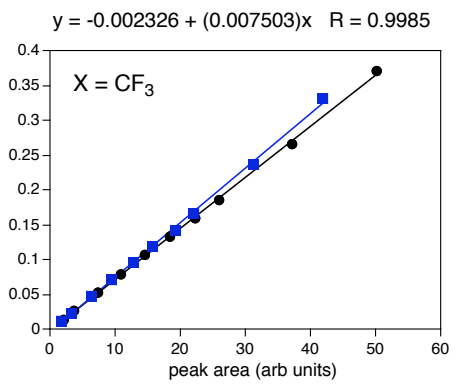

$y=-0.002057+(0.007322) x \quad R=0.99965$ $y=-0.004423+(0.007827) \times \quad R=0.99935$

$y=0.000293+(0.003476) x+\left(7.54 \cdot 10^{-6}\right) x^{2} \quad R=0.9997$

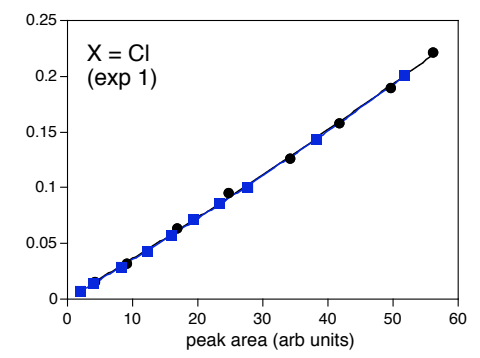

$y=0.0009215+(0.003493) x+\left(6.99 \cdot 10^{-6}\right) x^{2} R=0.99991$ $y=0.0001017+(0.003449) x+\left(8.08 \cdot 10^{-6}\right) x^{2} \quad R=0.99986$

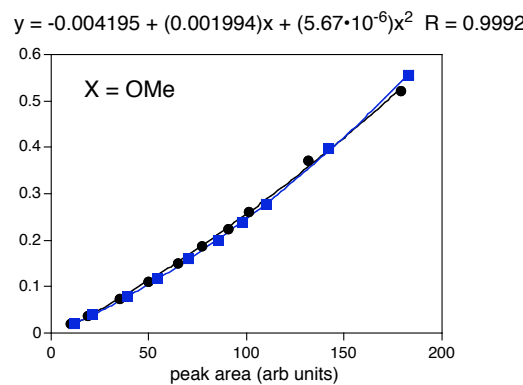

$y=-0.007348+(0.002208) x+\left(4.34 \cdot 10^{-6}\right) x^{2} \quad R=0.99926$ $y=-0.001507+(0.001795) x+\left(6.889 \cdot 10^{-6}\right) x^{2} R=0.99991$

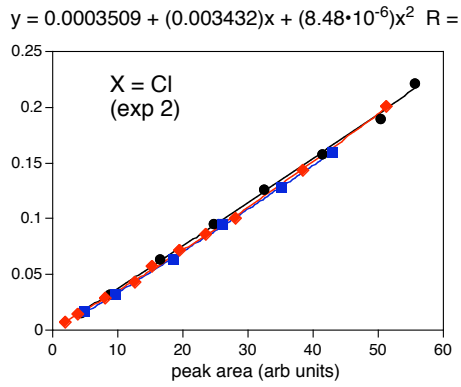

$y=-0.000356+(0.003743) x+\left(2.90 \cdot 10^{-6}\right) x^{2} \quad R=0.99924$ $y=-0.00194+(0.003506) x+\left(5.91 \cdot 10^{-6}\right) x^{2} \quad R=0.9998$ $y=0.001208+(0.003309) x+\left(1.10 \cdot 10^{-5}\right) x^{2} R=0.99961$ 
Figure S13 (Figure 3). Conditions: $2 \mathrm{mM}$ arylazide, $50 \mathrm{mM} \mathrm{PhCCH}, 0.1 \mathrm{mM} \mathrm{CuSO}_{4}, 0.2 \mathrm{mM} \mathrm{(BimH)}$, 1-benzyl-4-phenyl-1,2,3-triazole- $d 7$ internal standard, 4:1 $t-\mathrm{BuOH}: \mathrm{H}_{2} \mathrm{O}$, containing $25 \mathrm{mM}$ sodium ascorbate, $24{ }^{\circ} \mathrm{C}$. Each plot shows a set of three independent runs, set up at the same time with the same stock solutions. Two independent sets of experiments were performed for $p$-chlorophenylazide, as shown. For $\mathrm{X}=\mathrm{H}$ and $\mathrm{X}=\mathrm{CF}_{3}$, a small initial burst was ignored, and the rates obtained from fitting the data starting at $t=3$ and $6 \mathrm{~min}$, respectively.
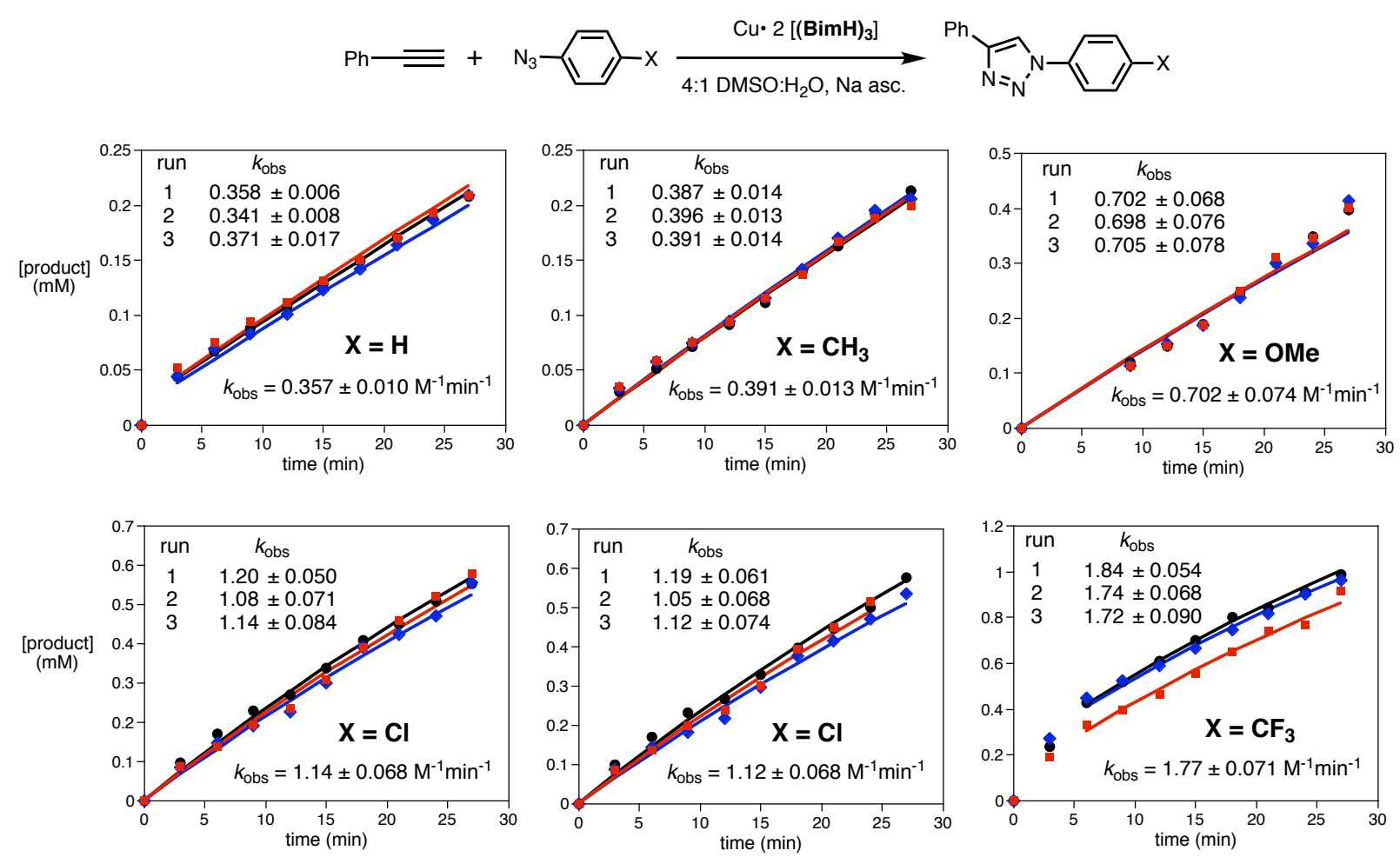

\section{References}

(1) Tanno, M.; Sueyoshi, S.; Kamiya, S. Chem. Pharm. Bull. 1982, 30, 3125-32.

(2) Andersen, J.; Madsen, U.; Bjorkling, F.; Liang, X. Synlett 2005, 2209-2213. 